\title{
MAPPING OF TECHNOLOGICAL INNOVATION LEVEL IN PROJECTS IN A UNIVERSITY OF AREQUIPA, PERU
}

\author{
J. Sulla-Torres', J. Esquicha Tejada1, K. Rosas Paredes', S. Moreno Rojas², Z. \\ Guerra De Castillo², A. Garcia-Carrillo ${ }^{3}$ \\ ${ }^{1}$ Universidad Católica de Santa María (PERU) \\ ${ }^{2}$ Universidad Tecnológica de Panamá (PANAMA) \\ ${ }^{3}$ Universitat Politècnica de Catalunya (SPAIN)
}

\begin{abstract}
Currently, the most developed societies invest in science and technology, due to the generation of knowledge and innovations, which apply scientific techniques and technological knowledge, fundamental for economic growth and social progress, for which it is necessary to periodically generate the mapping of the level of maturity of projects. The objective of the article is to map the maturity level of technological innovation within a University of Arequipa, Peru, for this, two tools have been used for its measurement: Technology Readiness Levels and the Minimum Viable Innovation System that allows validating the main hypotheses of value and build a final product. These tools were applied through a survey of the leaders of 186 projects financed by the University. The results of both measurements were compared and showed that out of the total of the responses obtained, they showed an average level of technological innovation, which is why it concludes that the technological innovation projects at the University of the case study do not go beyond a level 4 technology maturation, that is, an incipient innovation process.

Keywords: Mapping, Project, Technological Innovation, Level of Maturity of Technology, Minimum Viable Innovation System.
\end{abstract}

\section{INTRODUCTION}

Innovation is the process of converting new ideas into value, in the form of new products, services or ways of doing things.

The mapping and measurement of innovation in different companies have been carried out for several years [1] [2], likewise, the different countries have taken the importance and benefits of applying innovation in all fields [3] [4]. Innovation can occur in business models [5], however, in the field of technological innovation [6], the changes are faster and faster due to its progress, hence the importance of mapping the technological landscape [7] [8].

According to the World Economic Forum, it presents the context of innovation, its trends, research and analysis, dividing it into Systems Innovation, Government Innovation, Business Model Innovation, Innovation for Social Benefit and technological innovation.

There are different metrics to measure the level of technological innovation such as the Technology Readiness Levels (TRL) which are a type of measurement system used to assess the maturity level of a particular technology [9], The Minimum Viable Innovation System (MVIS) refers to the essential elements that enable a company to start creating a reliable and strategically focused innovation function. An MVIS will ensure that good ideas are encouraged, identified, shared, reviewed, prioritized, resourced, developed, rewarded, and celebrated. But it won't take years of work. [10].

Along these lines, the project "Construction and evaluation of innovative capacities in the academic sector" (CECIAC) is sponsored by the Ibero-American Program for Science and Technology for Development 
(CYTED), which aims to strengthen innovation management capacities (technological and nontechnological) with a pro-social orientation in Partner Universities of the GESIT Consortium.

In Arequipa, Peru, the Catholic University of Santa María, a member of CECIAC, has started several years ago with the development of research and innovation projects in its different areas, but did not yet have an innovation mapping, for this reason, it is that it became necessary to do it, based on the TRL and MVIS metrics.

The main objective of the article is to map the maturity level of technological innovation within a University of Arequipa, Peru. To achieve this objective, two tools have been used for its measurement: Technology Readiness Levels and the Minimum Viable Innovation System that allows validating the main value hypotheses and building a final product. After this introduction, this article is organized as follows: the review of related works is presented in section 2, the methodology is explained in section 3 , the results are shown in section 4, and finally the conclusions in section 5.

\section{RELATED WORK}

There is a wide variety of studies on innovation capabilities, considering the seven dimensions (learning, R\&D, resource allocation, production, marketing, organization and strategic planning capabilities) [11] [12], as well as technological development [13], and improving the performance of innovation lies in how to acquire the capacity for technological innovation with competitive advantages for those who apply it [14].

In relation to the impact of university-industry collaboration (UI), in the work of Shi, Wu and Fu (2020), they carry out a study on the efficiency of business innovation using a balanced panel of innovative companies. An evaluation of the innovation efficiency of companies shows that innovative companies do not show consistent performance throughout the two stages of the innovation process, namely the R\&D stage and the commercialization stage. The results demonstrate that UI collaboration affects innovation efficiency differently in the two stages, therefore, relevant policies may be required to facilitate user interface collaboration and its role in improving the efficiency of innovation. innovation in different stages [15].

Regarding Technology Readiness Levels used with the Academy, in the work of Liu, Subramanian and Hang (2020) they carried out a study on the transfer of technology from public research institutions to small (PRI) and medium-sized companies (SMEs) compared the information processing requirements with the Technology Readiness Levels of the technologies destined to be transferred from the PRI the information processing capacities with levels of preparation of the demand of the capacities that SMEs possess, finally presented an integrated framework for correspondence between source and receiver that facilitates the transfer of PRI-SMEs technology [16]. Dunhill, Thayer and Newton (2011) already analyzed the challenges of an organization that links industrial needs with academic research, emphasizing the importance of exploiting the results of research, using a system based on Technology Readiness Levels to facilitate the transfer process of technology [17].

In relation to the Minimum Viable Innovation System, in the work of Jaramillo, Montes and Aguirre (2016) they present the structuring and implementation of an ethical, sustainable and innovation-oriented marketing model that aims to structure a Minimum Viable Innovation System in each institution, through the transfer of skills and competences to the personnel linked to the institution [18]. Koscielniak (2016), shows the barriers to the implementation of companies' technological entrepreneurship solutions and the opportunities to support their development based on the Minimum Viable Innovation System [19].

In the work of Pietrovski et al (2020) he presents a management methodology for the Centers of Technological Innovation (ICT) in Higher Education Institutions (HEI), his methodological proposal had a qualitative approach and used a quantitative technique to make a diagnosis of ICT performance. The primary data collection included interviews with ICT managers in Brazil and at the Transfer Offices and Research Results Offices (RRTO) in Spain, and also a questionnaire applied to ICT representatives. Secondary data was collected from the documents. The results showed that ICTs are implemented or have actions that allow them to function, they have trained teams, however, the rotation of personnel is essential to satisfy the demands of ICTs. The main barriers to action refer to the allocation of vacancies, the selection criteria for ICT management and the recognition of researchers [20]. 
In the work of Pires M., Rita and Pies A. (2020) they analyzed the institutional profile of the Center for Technological Innovation (NIT) of the Federal University of Alagoas based on the New Legal Framework for Science, Technology and Innovation, such analysis allowed enhance the potential of ILS so that they can take more effective actions focused on the transfer of technologies for the generation of innovation that drives the country's socioeconomic development [21].

\section{METHODOLOGY}

The survey technique was used to measure the level of innovation of the projects carried out at the University. All the required information was recorded in two online questionnaires, one for TRL and one for MVIS.

The information collection was carried out during the even semester of 2019. The questionnaires were applied to the leaders of the projects, and the analysis and statistics were carried out in early 2020.

The projects reviewed are part of the competitive funds of the Catholic University of Santa María; Since 2015 , there have been six calls to windows that have brought together 186 projects.

The procedure followed by the projects for the award of funds is as follows:

- Announcement. The call process begins with the proposal and publication of the bases where the format, the qualification system, the means of presentation, the requirements at the curriculum level of the team of requesting researchers and the duration of the call are regulated.

A document of bases is developed that will govern each type of contest. For research projects, there are mostly three categories, and they differ from each other in the amount of budget allocated per winning project and in the professional requirements and the curricular evaluation of the participants.

In general, the call uses electronic media and social networks to disseminate and have greater reach.

- Presentation of the project. The presentation of the project is carried out only under the approved format and within the deadlines established in the bases of each contest. Exceptionally, you can opt for an extension of time that does not exceed one week. Depending on the conditions and problems that could arise in the application. There are two presentation platforms; the virtual platform with its own data entry system through fields, thus avoiding the use of paper, this being the official platform. Likewise, the presentation via email is available and it is applied only when the virtual platform presents any problem.

- Evaluation. Once the validity of the calls has ended, the projects received are closed and systematized.

A unique code is given to each project, the same one that is maintained throughout the process. The evaluation has two phases:

a. Eligibility: a committee is in charge of the eligibility that consists of reviewing the fulfillment of the requirements, with the validation of a list of requirements of each of the projects, in which the submitted documentation and the curriculum vitae of each of the projects are evaluated. The members of the submitted projects and contrasted with the requirements described in the contest rules, the technical-economic feasibility is also verified. Likewise, the projects presented will undergo a process of scientific integrity, using different tools, evidencing and analyzing the level of coincidences in order to establish their continuity for the next phase. If any failure or non-compliance is found, the project does not continue with the evaluation process.

b. Technical evaluation: For this second evaluation phase each of the projects is sent to two external peer evaluators according to the area and line of research, with experience in evaluating projects and scientific publications, who act as qualifying juries. Along with the project, the evaluation rubric set out in the bases is sent to them. In exceptional cases and when the evaluation of the evaluators is very different from each other, the inclusion of a third jury or deciding party is chosen, whose function is to issue a third evaluation, the latter being the one recorded within the total ranking.

- Qualification. For the qualification of the projects the Evaluators will be based on the following categories:

- Significance and relevance

- Methodology and agreement 
- Innovation

- Experience of the research team

- Award. The financial funds are awarded to the research projects in strict order of scoring. The funds will be awarded until the budget is exhausted. In case of remaining funds, they will be used for scientific research activities.

In the event that the difference in the scores assigned to a project by the external peer evaluators differs by $30 \%$ to more than the maximum score, a third evaluator will be used who will act as settler.

Subsequently, the ranking is prepared according to the average of the final grades obtained, which is validated by the Validation Committee for publication.

To be able to award the contest funds, it is required:

a) Carry out the reconciliation and approval of the Budget Operational Plan (POP).

b) Sign the Award Contract: Detailing Responsibilities, Benefits and Penalties as:

- Delivery of products according to Milestones.

- Incentive delivery to the research team: it will be delivered in two parts, upon approval of the 2nd milestone report and the final report.

- Penalty for failure to meet objectives: refund of the amount awarded and inclusion in the UCSM Registry of Ineligibles.

- Project development. The development of the project begins once the award contract is signed, this stage is linked to the monitoring and follow-up stage. Progress reports and financial reports are constantly requested on a monthly basis as an indication that the project is being executed.

- Monitoring. The Monitoring stage of the project consists of monitoring its development, which has the following phases:

Phase I: Presentation of the baseline by the research team and review by the project monitor according to FMP004 format.

The baseline is the first measurement of all the indicators contemplated in the design of the initial Application Project, and therefore, allows establishing the "starting point" of the project and technicalbudgetary honesty, in this sense; The accompaniment and monitoring of the milestones must be compared with the indicators of the Baseline to measure the progress, results and goals that were achieved or not, with the execution of the project. The baseline is the definition of before-after comparison parameters to identify and explain the effects of the project on dimensions and variables on which the project intends to influence.

Phase II: Presentation of the technical advances of research projects. The objective of which is to verify the committed indicators in each milestone, in the previous milestones and to verify the type of risk in the project's fulfillment. Likewise, resolve other people's situations arising in the fulfillment of the milestone. Here the monitor issues an opinion of the type Approved, Approved with reservations or Disapproved. FMP007 format.

Phase III: Presentation of progress of economic reports, which allows the financial analysis and verification of the support of expenses, that is, to verify the amounts programmed by item. Here the monitor issues an opinion of the type Approved, Approved with reservations or Disapproved.

- Close. The starting line concludes once the research team meets all the scheduled milestones, with the delivery of the final technical report, the final economic report, the deliverables required at the bases and the dissemination of the results in a public act.

Proceed with the issuance of the closing resolution by the competent authority.

In addition, the UCSM Office of Innovation and Development is in charge of the Entrepreneurship and Innovation Subsection, which has two areas:

- Research hotbed (E-quipu Santa María), complies with training students and graduates in their first investigations with topics related to the lines of research of the Professional Schools that UCSM has, in 
addition to being the pre-incubator for the University, which manages to strengthen its business idea until it has a functional prototype.

- Business Incubator (Innicia), its objective is to strengthen business models of incubations, providing the service of continuous training on issues related to the planning and development of business models, until the creation of your company.

The students and graduates trained in the Research Seedbed and in the Business Incubator are mostly the members of the research projects that are led by the university research professors.

Based on this procedure mentioned previously for the selection of projects financed by the University, the TRL and MVIS methodologies have been used to measure technological innovation.

For the construction of the TRL instrument it was used from the validity of the content that was proposed by NASA [9]

TRL has seven levels that are:

- Level 1: Basic research: the elementary principles are observed and referred

- Level 2: Applied research: the concept of technology and / or its application is formulated

- Level 3: Critical function, proof and establishment of the concept

- Level 4: Laboratory analysis of the process prototype

- Level 5: Laboratory analysis of integrated system

- Level 6: Verification of the prototype system

- Level 7: Demonstration of the integrated pilot system

- Level 8: The system incorporates commercial design

- Level 9: The system is ready for use at full scale

For the construction of the MVIS instrument, the validity of the content that was proposed by [10].

MVIS is made up of 4 phases:

- Define an innovation challenge

- Identify a concrete opportunity for innovation

- Select a pilot project for its execution

- Install the innovation system.

Which were raised in the evaluation instrument, according to the following sections:

Innovation Growth Plan:

1. The University has a defined Growth Strategy in innovation

2. Has the University identified areas of opportunity

Government and Control:

3. There is a government structure to promote innovation at the University

4. The University has Innovation Managers

Innovation Production System:

5. The University has a Portfolio of innovation projects

Leadership, Talent and Culture:

6. The University has groups or centers of researchers and innovators

7. The University has a communication strategy to disseminate the results of the innovation The following section presents the results obtained after applying the evaluation instruments. 


\section{RESULTS}

For the evaluation, two virtual questionnaires have been created for each evaluation metric, TRL and MVIS, from which the following responses have been obtained.

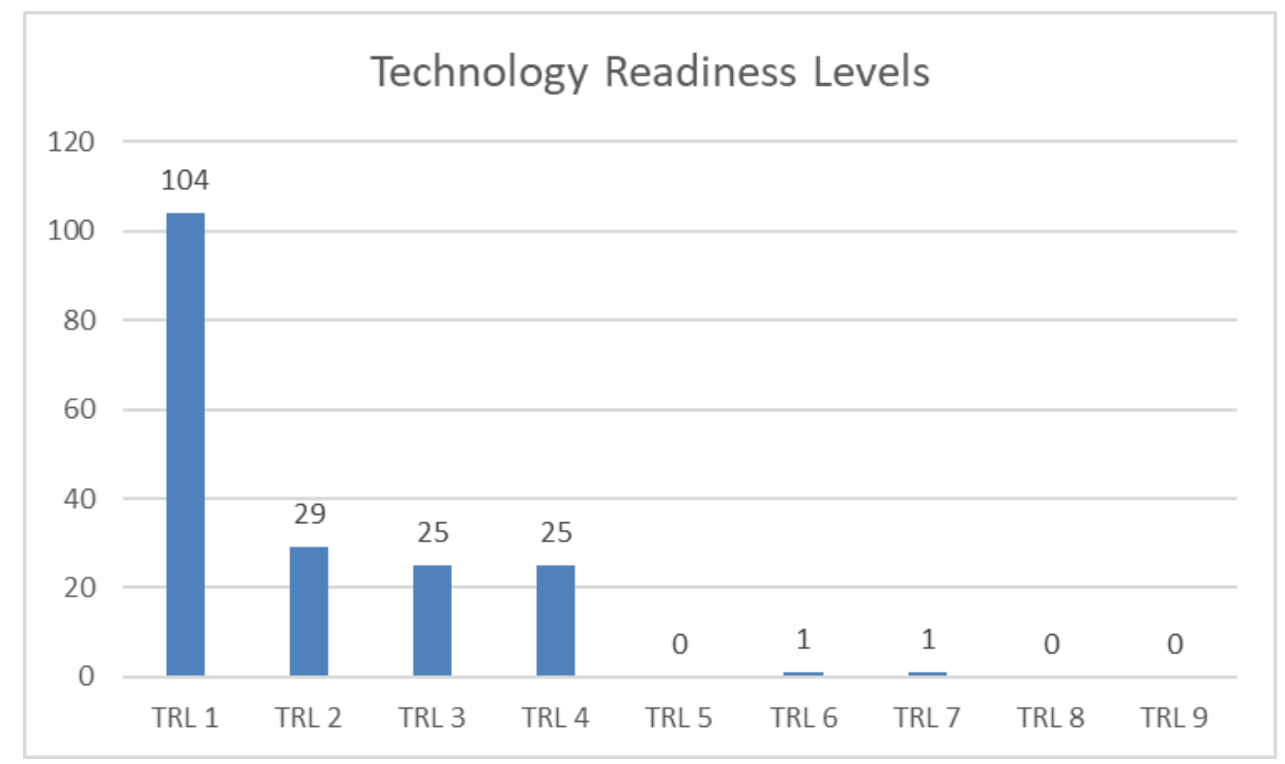

Figure 1. Result of the evaluation of the Technology Development Level (TRL) at UCSM

Figure 1 shows that of the 186 projects that the University of Arequipa, Peru has, there are $104(56.2 \%)$ projects that are in level 1, $29(15.7 \%)$ projects in level 2, $25(13.5 \%)$ at level 3 and 4 ; in addition, only one project at each level of 6 and $7(0.5 \%)$.

In the case of MVIS, an evaluation instrument was presented to leaders of the 186 projects, consisting of seven questions indicated in the previous section, with a scale of 1 to 5 where $1=$ Totally disagree and $5=$ Totally agree.

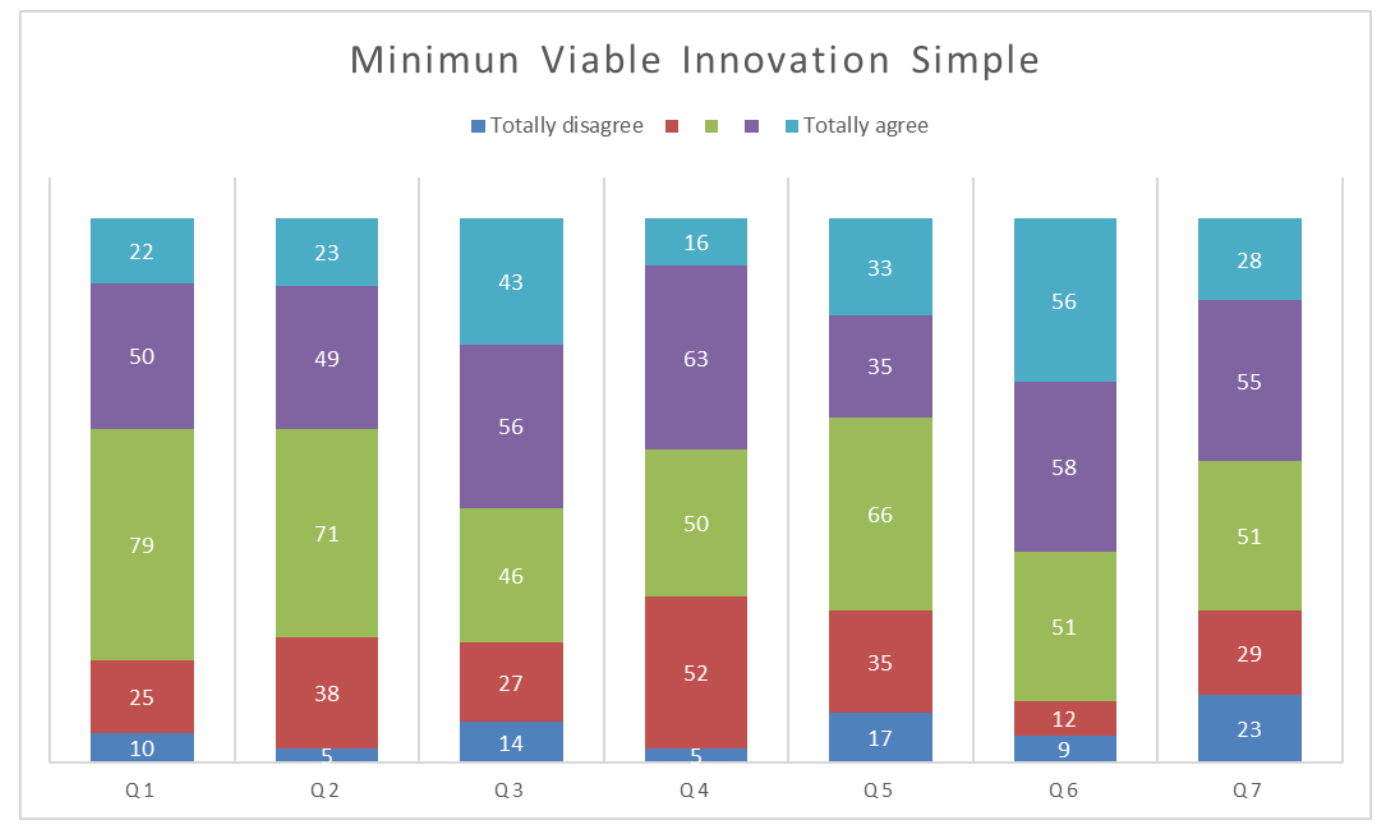

Figure 2. Result of the evaluation of the Minimum Viable Innovation Simple (MVIS) at UCSM. 
Figure 2 shows that of the 186 projects held at the University of study, 31.8\% (3rd section) of the projects are in the middle level, followed by a close $28.11 \%$ (2nd section). to the agreement, and $16.7 \%$ ( 1 st section) of Totally agree, obtaining a $76.61 \%$ higher average jointly.

\section{CONCLUSIONS}

Both methodologies used to measure technological innovation show that the University of Arequipa, Peru, has an average level of technological innovation, which was achieved thanks to the creation of the ViceRector's Office for Research since 2015, which allowed for the generation of competitive funds to gather 186 projects that take place at the University.

Using the TRL technological innovation measurement, it is evident that the University of Arequipa, Peru has a large number of projects that are in level $1(56.2 \%)$, but also a large number of projects in levels 2, 3 and $4(42.7 \%)$ which means that most projects do not go beyond a level 4 technology maturation, that is, an incipient innovation process.

With respect to MVIS, most of the projects show that they are $76.61 \%$ above the average, as they fully agree that the University has a growth plan in innovation, Government and Control, the Innovation Production System, as well such as leadership, talent and culture in innovation.

These results have allowed mapping the level of technological innovation in which the University is in the case study, so these results are of utmost importance for future decisions made by the University in relation to the development of projects, such as the approach It must be carried out with greater support for projects focused on technological innovation so that they can level up in the TRL, MVIS or other measurements used.

\section{ACKNOWLEDGEMENTS}

To the Universidad Católica de Santa María de Arequipa, Peru, for the financial support in the presentation of the Article and to CECIAC, because it is allowing the proposal to be developed with the scientific and academic operators of the collaborating countries of Argentina, Colombia, Chile, Ecuador, Guatemala, Panama, Peru and Spain, with the purpose of strengthening and promoting innovation in the region.

\section{REFERENCES}

[1] S. Brusoni, a Prencipe, and a Salter, "Mapping and Measuring Innovation in Project-based Firms," Current, 1998.

[2] D. Archibugi and M. Pianta, "Measuring technological change through patents and innovation surveys," Technovation, 1996.

[3] M. R. A. Rezk, H. H. Ibrahim, M. Tvaronavičienè, M. M. Sakr, and L. Piccinetti, "Measuring innovations in Egypt: case of industry," Entrep. Sustain. Issues, 2015.

[4] V. Boly, L. Morel, N. G. Assielou, and M. Camargo, "Evaluating innovative processes in french firms: Methodological proposition for firm innovation capacity evaluation," Res. Policy, 2014.

[5] T. Clauss, "Measuring business model innovation: conceptualization, scale development, and proof of performance," R D Manag., 2017.

[6] M. V. Türker, "A model proposal oriented to measure technological innovation capabilities of business firms - a research on automotive industry," Procedia - Soc. Behav. Sci., 2012.

[7] B. S. Aharonson and M. A. Schilling, "Mapping the technological landscape: Measuring technology distance, technological footprints, and technology evolution," Res. Policy, 2016.

[8] B. T. Kelly, D. Papanikolaou, A. Seru, and M. Taddy, "Measuring Technological Innovation over the Long Run," SSRN Electron. J., 2018.

[9] NASA, “Definition Of Technology Readiness Levels,” NASA Technol. Readiness Lev., 2015. 
[10] P. M. A. Siren, S. D. Anthony, and D. S. Duncan, "Build an Innovation Engine in 90 days," Harv. Bus. Rev., 2014.

[11] C. hsien Wang, I. yuan Lu, and C. bein Chen, "Evaluating firm technological innovation capability under uncertainty," Technovation, 2008.

[12] J. Liu, A. Baskaran, and S. Li, "Building technological-innovation-based strategic capabilities at firm level in China: A dynamic resource-based-view case study," Ind. Innov., 2009.

[13] S. Magistretti, C. Dell'Era, and R. Verganti, "Searching for the right application: A technology development review and research agenda," Technol. Forecast. Soc. Change, 2020.

[14] S. Sun and Q. Y. Tao, "The Relationship Between Technological Innovation Ability, Atmosphere and Innovation Performance," Int. J. Inf. Syst. SUPPLY Chain Manag., vol. 13, no. 2, pp. 47-58, 2020.

[15] X. Shi, Y. Wu, and D. Fu, "Does University-Industry collaboration improve innovation efficiency? Evidence from Chinese Firms $\diamond$," Econ. Model., 2020.

[16] H. Y. Liu, A. M. Subramanian, and C. C. Hang, "Marrying the Best of Both Worlds: An Integrated Framework for Matching Technology Transfer Sources and Recipients," IEEE Trans. Eng. Manag., 2020.

[17] A. K. Dunhill, P. J. Thayer, and K. Newton, "The advantages and challenges of linking industrial needs with academic research," Insight Non-Destructive Test. Cond. Monit., 2011.

[18] C. A. A. Jaramillo and L. de Innovación, "Modelo de comercialización ético, sostenible y en clave de innovación," in X Workshop de la Red EmprendeSUR 2016, 2016, p. 48.

[19] H. Kościelniak, "Constraints and barriers to technology entrepreneurship of enterprises in empirical studies," Organ. i Zarz. Kwart. Nauk., 2016.

[20] E. F. Pietrovski, D. R. dos Reis, J. L. Kovaleki, and V. I. Rasoto, "METHODOLOGY OF MANAGEMENT FOR THE TECHNOLOGICAL INNOVATION CENTERS," Rev. GEINTECGESTAO Inov. E Tecnol., vol. 10, no. 1, pp. 5234-5251, 2020.

[21] M. C. Ferreira Silva Pires, L. P. Santa Rita, and A. C. Santos Pires, "Profile of the technological innovation nucleus in innovation management: a study at the Universidade Federal de Alagoas," NAVUS-REVISTA Gest. E Tecnol., 2020. 